\title{
Clustered Planarity Testing Revisited
}

\author{
Radoslav Fulek $^{1,4, \star}$, Jan Kynčl ${ }^{1, \star \star}$, Igor Malinović ${ }^{2}$, and Dömötör Pálvölgyi ${ }^{3, \star \star \star}$ \\ 1 Department of Applied Mathematics and Institute for Theoretical Computer Science, \\ Faculty of Mathematics and Physics, Charles University, Malostranské nám. 25, \\ 11800 Praha 1, Czech Republic \\ radoslav.fulek@gmail.com, kyncl@kam.mff.cuni.cz \\ 2 Faculté Informatique et Communications, École Polytechnique Fédérale de Lausanne, \\ 1015 Lausanne, Switzerland \\ igor.malinovic@epfl.ch \\ ${ }^{3}$ Institute of Mathematics, Eötvös University, Pázmány Péter sétány 1/C, \\ H-1117 Budapest, Hungary \\ domotorp@gmail.com \\ 4 Department of Industrial Engineering and Operations Research, Columbia University, \\ New York City, NY, USA
}

\begin{abstract}
The Hanani-Tutte theorem is a classical result proved for the first time in the 1930s that characterizes planar graphs as graphs that admit a drawing in the plane in which every pair of edges not sharing a vertex cross an even number of times. We generalize this classical result to clustered graphs with two disjoint clusters, and show that a straightforward extension of our result to flat clustered graphs with three or more disjoint clusters is not possible.

We also give a new and short proof for a related result by Di Battista and Frati based on the matroid intersection algorithm.
\end{abstract}

\section{Introduction}

Investigation of graph planarity can be traced back to the 1930s and developments accomplished at that time by Hanani [21], Kuratowski [26], Whitney [38] and others. Forty years later, with the advent of computing machinery, a linear time algorithm for graph planarity was discovered [23]. Nowadays, a polynomial time algorithm for testing whether a graph admits a crossing-free drawing in the plane could almost be considered a folklore result.

Nevertheless, many variants of planarity are still only poorly understood. As a consequence of this state of affairs, the corresponding decision problem for these variants has neither been shown to be polynomial nor NP-hard. Clustered planarity is one of

* The author gratefully acknowledges support from the Swiss National Science Foundation Grant No. 200021-125287/1 and ESF Eurogiga project GraDR as GAČR GIG/11/E023.

** Supported by the ESF Eurogiga project GraDR as GAČR GIG/11/E023 and by the grant SVV-2013-267313 (Discrete Models and Algorithms).

*** Supported by Hungarian National Science Fund (OTKA), under grant PD 104386 and under grant NN 102029 (EUROGIGA project GraDR 10-EuroGIGA-OP-003) and the János Bolyai Research Scholarship of the Hungarian Academy of Sciences. 
the most prominent [5] of such planarity notions. Roughly speaking, an instance of this problem is a graph whose vertices are partitioned into clusters. The question is, then, whether the graph can be drawn in the plane so that the vertices from the same cluster belong to the same region and no edge crosses the boundary of a particular region more than once. The aim of the present work is to offer novel perspectives on clustered planarity, which seem to be worth pursuing in order to better our understanding of the problem.

More precisely, a clustered graph is a pair $(G, T)$ where $G=(V, E)$ is a graph and $T$ is a rooted tree whose set of leaves is the set of vertices of $G$. The non-leaf vertices of $T$ represent the clusters. For $\nu \in V(T)$, let $T_{\nu}$ denote the subtree of $T$ rooted at $\nu$. The cluster $V(\nu)$ is the set of leaves of $T_{\nu}$. The subgraph of $G$ induced by $V(\nu)$ is denoted by $G(\nu)$.

A drawing of $G$ is a representation of $G$ in the plane where every vertex is represented by a unique point and every edge $e=u v$ is represented by a simple arc joining the two points that represent $u$ and $v$. If it leads to no confusion, we do not distinguish between a vertex or an edge and its representation in the drawing and we use the words "vertex" and "edge" in both contexts. We assume that in a drawing no edge passes through a vertex, no two edges touch and every pair of edges cross in finitely many points. A drawing of a graph is an embedding if no two edges cross.

A clustered graph $(G, T)$ is clustered planar (or briefly $c$-planar) if $G$ has an embedding in the plane such that

(i) for every $\nu \in V(T)$, there is a topological disc $d(\nu)$ containing all the leaves of $T_{\nu}$ and no other vertices of $G$ such that if $\mu \in T_{\nu}$ then $d(\mu) \subseteq d(\nu)$;

(ii) if $\mu_{1}$ and $\mu_{2}$ are children of $\nu$ in $T$, then $d\left(\mu_{1}\right)$ and $d\left(\mu_{2}\right)$ are internally disjoint;

(iii) every edge of $G$ intersects the boundary of $d(\nu)$ at most once for every $\nu \in V(T)$.

A clustered drawing (or embedding) of a clustered graph $(G, T)$ is a drawing (or embedding, respectively) of $G$ satisfying (i)-(iii). See Fig. 1 for an illustration. We will be using the word "cluster" for both the topological disc $d(\nu)$ and the subset of vertices $V(\nu)$.

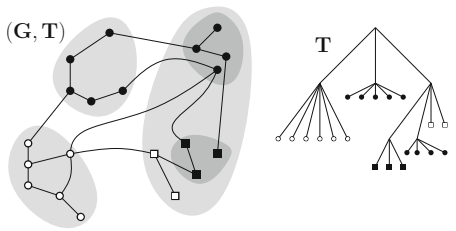

(a)

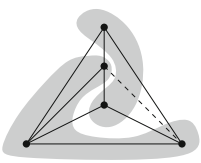

(b)

Fig. 1. (a) A clustered embedding of a clustered graph $(G, T)$ and its tree $T$; (b) A clustered graph with two non-trivial clusters, which is not c-planar

The notion of clustered planarity was introduced in the work of Feng, Cohen and Eades [13 14] under the name of c-planarity and a similar problem was considered 
already in [28]. Since then an efficient algorithm for c-planarity testing or embedding has been discovered only in some special cases. The general problem whether the cplanarity of a clustered graph can be tested in polynomial time is wide open, already when we restrict ourselves to three pairwise disjoint clusters and the case when the embedding of $G$ is a part of the input!

A clustered graph $(G, T)$ is $c$-connected if every cluster of $(G, T)$ induces a connected subgraph. In order to test a c-connected clustered graph $(G, T)$ for c-planarity, it is enough to test whether there exists an embedding of $G$ in which for every $\nu \in V(T)$ all vertices $u \in V(G)$ such that $u \notin V(\nu)$ are drawn in a single face of the subgraph $G(\nu)$ [14]. Cortese et al. [6] gave a structural characterization of c-planarity for c-connected clustered graphs and provided a linear-time algorithm. The extended abstract of Gutwenger et al. [19] contains an efficient algorithm in a more general case of almost connected clustered graphs, which can be also used in the case of two clusters. Biedl [2] is usually credited for giving the first polynomial time algorithm for c-planarity with two clusters, including the case of straight-line or $y$-monotone drawing. An alternative approach to the problem is given in [22]. On the other hand, only very little is known in the case of three clusters, where already clustered cycles are non-trivial to test for c-planarity [7] in a polynomial time.

The Hanani-Tutte theorem [2137] is a classical result that provides an algebraic characterization of planarity with interesting (and not only algorithmic) consequences; see Section 2. The (strong) Hanani-Tutte theorem says that a graph is planar as soon as it can be drawn in the plane so that no pair of independent edges crosses an odd number of times. Moreover, its variant known as the weak Hanani-Tutte theorem [3|30|33] states that if we have a drawing $\mathcal{D}$ of a graph $G$ where every pair of edges cross an even number of times then $G$ has an embedding that preserves the cyclic order of edges at vertices from $\mathcal{D}$. Note that the weak variant does not directly follow from the strong Hanani-Tutte theorem. For sub-cubic graphs, the weak variant implies the strong variant. Other variants of the Hanani-Tutte theorem were proved for surfaces of higher genus [32|34], $x$-monotone drawings [17/31], partially embedded planar graphs, and several special cases of simultaneously embedded planar graphs [36]. See [35] for a (not too recent) survey on applications of the Hanani-Tutte theorem and related results.

We prove a variant of the Hanani-Tutte theorem for clustered graphs consisting only of two non-trivial clusters forming a partition of the vertex set. Similarly, as in the case of other variants of the Hanani-Tutte theorem, as a byproduct of our result, we immediately obtain a polynomial-time algorithm based on linear algebra for c-planarity testing in the corresponding case. The downside is that the running time of the algorithm is in $O\left(|V(G)|^{2 \omega}\right)$, where $O\left(n^{\omega}\right)$ is the complexity of multiplication of square $n \times n$ matrices; see Section 2 The best current algorithms for matrix multiplication give $\omega<2.3729$ [1839]. This fact does not make our work less interesting, since the purpose of our results lies more in theoretical foundations than in its immediate consequences. Also the worst case running time analysis often gives an unfair perspective on the performance of algebraic algorithms, e.g., the simplex method.

We remark that there exist more efficient algorithms for planarity testing using the Hanani-Tutte theorem such as the one in [9]10], which runs in linear time, see also [35. Section 1.4.1]. Moreover, in the case of $x$-monotone drawings a computation study [4] 
showed that the Hanani-Tutte approach [17] performs really well in practice. This should come as no surprise, since Hanani-Tutte theory seems to provide solid theoretical foundations for graph planarity that brings together its combinatorial, algebraic, and computational aspects [36].

Notation. In the present paper we assume that $G=(V, E)$ is a (multi)graph. We use a shorthand notation $G-v$ and $G \cup E^{\prime}$ for $(V \backslash\{v\}, E \backslash\{v w \mid v w \in E\})$, and $\left(V, E \cup E^{\prime}\right)$, respectively. The rotation at a vertex $v$ is the clockwise cyclic order of the end pieces of edges incident to $v$. The rotation system of a graph is the set of rotations at all its vertices. We say that two embeddings of a graph are the same if they have the same rotation system up to switching the orientations of all the rotations simultaneously. We say that a pair of edges in a graph are independent if they do not share a vertex. An edge in a drawing is even if it crosses every other edge an even number of times. A drawing of a graph is even if all edges are even.

Hanani-Tutte for Clustered Graphs. A clustered graph $(G, T)$ is two-clustered if the root of $T$ has exactly two children and only leaves as grandchildren. In other words, a two-clustered graph has exactly two non-trivial clusters, which form a partition of the vertex set.

We extend the strong version of the Hanani-Tutte theorem as follows. A drawing of a graph is independently even if every pair of independent edges in the drawing cross an even number of times.

Theorem 1. If a two-clustered graph $(G, T)$ admits an independently even clustered drawing then $(G, T)$ is c-planar.

The weak variant of Theorem 1 is a special case of the result obtained recently by the first author [15]. On the other hand, we exhibit examples of clustered graphs with more than two disjoint clusters that are not c-planar, but admit an even clustered drawing.

Theorem 2. For every $r \geq 3$ there exists a flat clustered cycle with $r$ clusters that is not c-planar and admits an even clustered drawing.

Gutwenger et al. [20] recently showed that by using the reduction from [36] our counter-examples can be turned into counter-examples for [36, Conjecture 1.2] and for a variant of the Hanani-Tutte theorem for two simultaneously embedded planar graphs [36. Conjecture 6.20]. Our counter-examples show that a straightforward extension of Theorem 1 or its weak variant to flat clustered graphs with more than two clusters is not possible. Nevertheless, interesting extensions are still possible, e.g., in the c-connected case, as shown in the full version of this extended abstract [16], or for strip clustered graphs [15].

A clustered graph $(G, T)$ is flat if no non-root cluster of $(G, T)$ has a non-trivial subcluster; that is, if every root-leaf path in $T$ has at most three vertices. A pair $(\mathcal{D}(G), T)$ is an embedded clustered graph if $(G, T)$ is a clustered graph and $\mathcal{D}(G)$ is an embedding of $G$ in the plane, not necessarily a clustered embedding. The embedded clustered graph $(\mathcal{D}(G), T)$ is $c$-planar if it can be extended to a clustered embedding of $(G, T)$, by choosing a topological disc for each cluster. 
We give an alternative polynomial time algorithm for deciding c-planarity of embedded flat clustered graphs with small faces, reproving a result of Di Battista and Frati [11]. Our algorithm is based on the matroid intersection theorem. Its running time is $O\left(|V(G)|^{3.5}\right)$ by [8], so it does not outperform the linear algorithm from [11], and similarly as for our other results we see its purpose more in mathematical foundations than in giving an efficient algorithm. We find it quite surprising that by using completely different techniques we obtained an algorithm for exactly the same case. Our approach is very similar to a technique used [25] for deciding the global connectivity of switch graphs.

Theorem 3. [11] Let $G$ denote an embedded planar graph such that all its faces are incident to at most five vertices. Let $(G, T)$ denote a flat clustered graph. We can decide in polynomial time whether $(G, T)$ admits a c-planar embedding, in which $G$ keeps its given embedding.

The rest of the paper is organized as follows. In Section 2 we describe an algorithm for c-planarity testing of clustered graphs belonging to classes for which the corresponding variant of the strong Hanani-Tutte theorem holds. In Section 3 , we prove Theorem 1 In Section 4 we prove Theorem 2 In Section 5 we prove Theorem 3 . We conclude with some remarks in Section 6 .

\section{Algorithm}

Let $(G, T)$ belong to a class of clustered graph for which the corresponding variant of the strong Hanani-Tutte theorem holds, i.e., an independently even clustered drawing of $(G, T)$ implies that $(G, T)$ is c-planar.

Our algorithm for c-planarity testing is an adaption of the algorithm for planarity testing from [35, Section 1.4.2]. The algorithm tests whether we can continuously deform a given clustered drawing $\mathcal{D}$ of $(G, T)$ into an independently even clustered drawing $\mathcal{D}^{\prime}$ of $(G, T)$. By the corresponding variant of the strong Hanani-Tutte theorem, the existence of such a drawing is equivalent to c-planarity of $(G, T)$.

During the deformation the parity of crossings between a pair of edges is affected only when an edge $e$ passes over a vertex $v$, in which case we change the parity of crossings of $e$ with all the edges adjacent to $v$. We call such an event an edge-vertex switch. Note that every edge-vertex switch can be performed independently of others, for any initial drawing: we can always deform a given edge to pass close to a given vertex, while introducing new crossings only in pairs. Thus, for our purpose the continuous deformation of $\mathcal{D}$ can be represented by a set $S$ of edge-vertex switches. In $S$, an edge-vertex switch of an edge $e$ with a vertex $v$ is represented as the ordered pair $(e, v)$.

A drawing of $(G, T)$ can then be represented as a vector $\mathbf{v} \in \mathbb{Z}_{2}^{M}$, where $M$ denotes the number of unordered pairs of independent edges. The component of $\mathbf{v}$ corresponding to a pair $\{e, f\}$ is 1 if $e$ and $f$ cross an odd number of times and 0 otherwise. An edge-vertex switch $(e, v)$ is represented as a vector $\mathbf{w}_{(e, v)} \in \mathbb{Z}_{2}^{M}$ such that its only components equal to 1 are those indexed by pairs $\{e, f\}$ where $f$ is incident to $v$. The set of all drawings that can be obtained from $(G, T)$ by the switches from $S$ then corresponds to an affine subspace $\mathbf{v}+W$, where $W$ is the subspace generated by the set 
$\left\{\mathbf{w}_{(e, v)} ;(e, v) \in S\right\}$. The algorithm tests whether $\mathbf{0} \in \mathbf{v}+W$, which is equivalent to the solvability of a system of linear equations over $\mathbb{Z}_{2}$.

The difference between the original algorithm for planarity testing and our version for $c$-planarity testing is the following. To keep the drawing of $(G, T)$ clustered after every deformation, for every edge $e=v_{1} v_{2}$, we allow only those edge-vertex switches $(e, v)$ such that $v$ is a child of some vertex of the shortest path between $v_{1}$ and $v_{2}$ in $T$. We also include edge-cluster switches $(e, C)$, where $C$ is a child of some vertex of the shortest path between $v_{1}$ and $v_{2}$ in $T$, that move $e$ over all vertices of $C$ simultaneously. The corresponding vector $\mathbf{w}_{(e, C)}$ is the sum of all $\mathbf{w}_{(e, v)}$ for $v \in C$. Therefore, the set of allowed switches generates a subspace $W_{c}$ of $W$. Our algorithm then tests whether $\mathbf{0} \in \mathbf{v}+W_{c}$.

Before running the algorithm, we first remove any loops and parallel edges and check whether $\left|E\left(G^{\prime}\right)\right|<3\left|V\left(G^{\prime}\right)\right|$ for the resulting graph $G^{\prime}$. Then we run our algorithm on $\left(G^{\prime}, T\right)$. This means solving a system of $O\left(\left|E\left(G^{\prime}\right)\right|\left|V\left(G^{\prime}\right)\right|\right)=O\left(|V(G)|^{2}\right)$ linear equations in $O\left(\left|E\left(G^{\prime}\right)\right|^{2}\right)=O\left(|V(G)|^{2}\right)$ variables. This can be performed in $O\left(|V(G)|^{2 \omega}\right) \leq$ $O\left(|V(G)|^{4.752}\right)$ time using the algorithm by Ibarra, Moran and Hui [24].

\section{Two Clusters}

Let $(G, T)$ be a two-clustered graph. Let $A$ and $B$ denote the two clusters of $(G, T)$ forming a partition of $V=V(G)$. For a subset $V^{\prime} \subseteq V$, let $G\left[V^{\prime}\right]$ denote the subgraph of $G$ induced by $V^{\prime}$. By the assumption of Theorem 1 and the strong Hanani-Tutte theorem, $G$ has an embedding. However, in this embedding, $G[B]$ does not have to be contained in a single face of $G[A]$ and vice-versa. Hence, we cannot guarantee that a clustered embedding of $(G, T)$ exists so easily.

For an induced subgraph $H$ of $G$, the boundary of $H$ is the set of vertices in $H$ that have a neighbor in $G-H$. We say that an embedding $\mathcal{D}(H)$ of $H$ is exposed if all vertices from the boundary of $H$ are incident to the outer face of $\mathcal{D}(H)$.

The following lemma is an easy consequence of the strong Hanani-Tutte theorem. It helps us to find an exposed embedding of each connected component $X$ of $G[A]$ and $G[B]$. Later in the proof of Theorem 1 this allows us to remove non-essential parts of each such component $X$ and concentrate only on a subgraph $G^{\prime}$ of $G$ in which both $G[A]$ and $G[B]$ are outerplanar.

Lemma 1. Suppose that $(G, T)$ admits an independently even clustered drawing. Then every connected component of $G[A] \cup G[B]$ admits an exposed embedding.

Our proof of Theorem 1 proceeds by reducing the problem to an application of the following lemma.

Lemma 2. Let $(G, T)$ denote a two-clustered bipartite graph in which the two nontrivial clusters induce independent sets. If $G$ admits an even drawing then $(G, T)$ is c-planar. Moreover, there exists a clustered embedding of $(G, T)$ with the same rotation system as in the given even drawing of $G$. 


\subsection{Proof of Theorem 1}

The proof is inspired by the proof of the strong Hanani-Tutte theorem from [33] and its outline is as follows. First we obtain a subgraph $G^{\prime}$ of $G$ containing the boundary of each component of $G[A]$ and $G[B]$ and such that each of $G^{\prime}[A]$ and $G^{\prime}[B]$ is a cactus forest, that is, a graph where every two cycles are edge disjoint. Equivalently, a cactus forest is a graph with no subdivision of $K_{4}-e$. A connected component of a cactus forest is called a cactus.

Then we apply the strong Hanani-Tutte theorem on a graph which is constructed from $G^{\prime}$ by turning all cycles in $G^{\prime}[A]$ and $G^{\prime}[B]$ into wheels, and by splitting certain vertices of $G^{\prime}$ into edges. The wheels in $G^{\prime}$ guarantee that everything that has been removed from $G$ in order to obtain $G^{\prime}$ can be inserted back.

Let $X_{1}, \ldots, X_{k}$ denote the connected components of $G[A]$ and $G[B]$. By Lemma 1 we find an exposed embedding $\mathcal{D}\left(X_{i}\right)$ of each $X_{i}$. Let $X_{i}^{\prime}$ denote the subgraph of $X_{i}$ obtained by deleting from $X_{i}$ all the vertices and edges not incident to the outer face of $\mathcal{D}\left(X_{i}\right)$. Observe that $X_{i}^{\prime}$ is a cactus.

Let $G^{\prime}=\left(\bigcup_{i=1}^{k} X_{i}^{\prime}\right)+E(A, B)$. That is, $G^{\prime}$ is subgraph of $G$ that consists of all $X_{i}^{\prime}$-s and all edges between the two clusters. Let $\mathcal{D}^{\prime}$ denote the drawing of $G^{\prime}$ obtained from the initial independently even drawing of $G$ by deleting the edges and vertices of $G$ not belonging to $G^{\prime}$. Thus, $\mathcal{D}^{\prime}$ is independently even.

In what follows we process the cycles of $G^{\prime}[A]$ and $G^{\prime}[B]$ one by one. We will be modifying $G^{\prime}$ and therefore also the drawing $\mathcal{D}^{\prime}$. At each stage of this process some cycles in $G^{\prime}[A]$ and $G^{\prime}[B]$ will be labeled as processed and the rest will be labeled as unprocessed. We will maintain the property that all processed cycles are vertex disjoint and that all their edges are even. We start with all the cycles in $G^{\prime}[A]$ and $G^{\prime}[B]$ being labeled as unprocessed. Let $C$ denote an unprocessed cycle in $G^{\prime}[A]$. For cycles in $G^{\prime}[B]$, the procedure is analogous. We consider two cases.

a) $C$ Shares no Vertex with an Already Processed Cycle. We two-color the connected regions in the complement of $C$ so that two regions sharing a non-trivial part of the boundary receive opposite colors. We say that a point not lying on $C$ is "outside" of $C$ if it is contained in the region with the same color as the unbounded region. Otherwise, such a point is "inside" of $C$.

We locally modify the drawing $\mathcal{D}^{\prime}$ at the vertices of $C$ so that all the edges of $C$ cross every other edge an even number of times [33]. Since $\mathcal{D}^{\prime}$ is a clustered drawing of $G^{\prime}$, all vertices of $B$ are "outside" of $C$. Therefore, every path joining $C$ with a vertex in $B$ internally vertex disjoint from $C$ is attached to its endpoint on $C$ from the "outside" of $C$.

Now we fill the cycle $C$ with a wheel. More precisely, we add a vertex $v_{C}$ into $A$ and place it very close to an arbitrary vertex of $C$ "inside" of $C$. We connect $v_{C}$ with all the vertices of $C$ by edges that closely follow the closed curve representing $C$ either from the left or from the right, and attach to their endpoints on $C$ from "inside". Portions of these new edges may lie "outside" of $C$ due to self-crossings of $C$, but not in the neighborhood of vertices of $C$. Therefore, the new edges can introduce an odd crossing pair only with an edge $e$ attached to a vertex $v$ of $C$ from the "inside" of $C$.

Since $G^{\prime}[A]$ is a cactus forest, it follows that such a vertex $v$ is a cut vertex in $G^{\prime}[A]$ and that the endpoint of $e$ different from $v$ belongs to a connected component $K$ of 
$G^{\prime}[A]-v$, which is also a connected component of $G^{\prime}-v$. Thus, we shrink the drawing of $G^{\prime}[V(K) \cup v]$ so that $G^{\prime}[V(K) \cup v]$ is drawn very close to $v$ and none of its edges crosses an edge in the rest of the graph. In particular, by shrinking $G^{\prime}[V(K) \cup v]$ we do not introduce a pair of edges crossing an odd number of times. We label all the cycles in $G^{\prime}[V(K) \cup v]$ as processed. By repeating this for all the troublesome cut-vertices of $C$ we modify $\mathcal{D}^{\prime}$ so that none of the edges incident to $v_{C}$ crosses another edge an odd number of times. Finally, we label $C$ as processed.

b) $C$ Shares a Vertex with an Already Processed Cycle. Let $v$ be a vertex on $C$ belonging to an already processed cycle $C_{p}$. Since processed cycles are vertex disjoint, the cycle $C_{p}$ is unique. Since the edges of $C_{p}$ are even, the edges $v_{1} v$ and $v_{2} v$ of $C_{p}$ adjacent to $v$ are attached to $v$ both from the "inside" or both from the "outside" of $C$. Suppose the latter. (The other case is analogous.) We split the vertex $v$ by replacing it with two new vertices $v^{\prime}$ and $v^{\prime \prime}$ connected by an edge. Every edge $u v$ attaching to $v$ from the "outside" of $C$ is replaced by an edge $u v^{\prime}$ (including the edges $v_{1} v$ and $v_{2} v$ ). All other edges $u v$ are replaced by an edge $u v^{\prime \prime}$. The cycle that is obtained from $C_{p}$ by replacing $v$ with $v^{\prime}$ is then labeled as processed. Note that we can do such vertexsplitting in $\mathcal{D}^{\prime}$ without introducing any pair of edges crossing an odd number of times by drawing $v^{\prime}$ and $v^{\prime \prime}$ very close to $v$. After performing all necessary vertex splits for vertices of $C$, we may apply the procedure in case a) to the modified cycle $C$.

It is easy to see that the algorithm terminates after a finite number of steps a) or b) with all cycles processed. Let $G^{\prime \prime}$ denote the graph we obtain from $G^{\prime}$ after processing all the cycles of $G^{\prime}[A]$ and $G^{\prime}[B]$. By applying the strong Hanani-Tutte theorem on $G^{\prime \prime}$ we obtain an embedding which can be easily modified so that the only vertices of $G^{\prime \prime}$ not incident to the outer face of $G^{\prime \prime}[A]$ or $G^{\prime \prime}[B]$ are the vertices $v_{C}$ that form the centers of the wheels. In particular, $G^{\prime \prime}[A]$ is drawn in the outer face of $G^{\prime \prime}[B]$ and viceversa. In the resulting embedding we delete all the vertices $v_{C}$ and contract the edges between the pairs of vertices $v^{\prime}, v^{\prime \prime}$ that were obtained by vertex-splits.

Thus, we obtain an embedding of $G^{\prime}$ in which for every component $X$ of $G^{\prime}[A] \cup$ $G^{\prime}[B]$, all vertices of $G^{\prime}-X$ are drawn in the outer face of $X$. By inserting the removed parts of $G$ back to $G^{\prime}$ we obtain an embedding of $G$ in which for every component $X$ of $G[A] \cup G[B]$, all vertices of $G-X$ are drawn in the outer face of $X$. The theorem follows by contracting each component of $G[A] \cup G[B]$ to a point and applying Lemma2

\section{Proof of Theorem 2}

In this section we construct a family of even clustered drawings of flat clustered cycles on more than two clusters that are not clustered planar. Thus, the straightforward generalization of the Hanani-Tutte theorem for graphs with three or more clusters is not possible. For any $r \geq 3$ and any odd $k \geq 3$, our counterexample is a clustered cycle with $k r$ vertices and $r$ clusters. In our clustered drawing the clusters are drawn as regions bounded by a pair of rays emanating from the same vertex $p$. We call $p$ the center of our drawing.

Topologically our construction is equivalent to a cylindrical drawing, where $c$ clusters are separated by vertical lines. For every odd integer $k>0$, we can describe the curve representing the cycle analytically as a height function $f(\alpha)=\sin \left(\frac{r k+1}{k} \alpha\right)$ on 
a standing cylinder taking the angle as the parameter. The vertices of the cycle are at $\left(i \frac{2 k}{r k+1} \pi, 0\right)$, where $i=0, \ldots, r k-1$, and the lines separating clusters at $\frac{2 k i+1}{r k+1} \pi$, for $i=0, \ldots r-1$, see Fig. 2] By [7], for any clustered drawing of any of our examples, the curve representing the cycle has winding number $k$ around $p$, and therefore, it is not c-planar when $k>1$.

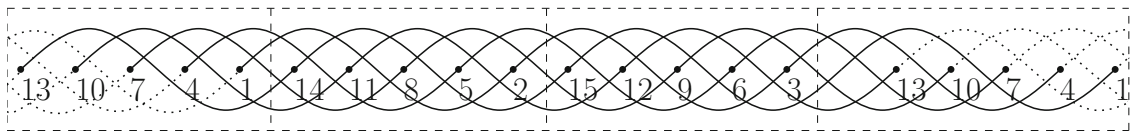

Fig. 2. A counter-example to the variant of the Hanani-Tutte theorem with parameters $r=3$ and $k=5$, and hence, the underlying graph is a cycle on 15 vertices. The vertices are labeled by positive integers in correspondence with their appearance on the cycle. The leftmost and the rightmost cluster need to be identified in the actual cylindrical drawing.

\section{Small Faces}

This section reproves a result of Di Battista and Frati [11] that c-planarity can be decided in polynomial time for embedded flat clustered graphs if all faces are incident to at most five vertices. Our approach seems quite different from theirs, as we use (a corollary of) the matroid intersection theorem [12 27], which says that the largest common independent set of two matroids can be found in polynomial time. See e.g. [29] for further references.

In this section, we will use a shorthand notation $(G, T)$ instead of $(\mathcal{D}(G), T)$ for an embedded clustered graph. Let $(G, T)$ be a embedded flat clustered graph. A saturator of $(G, T)$ is a subset $F$ of $\left(\begin{array}{c}V \\ 2\end{array}\right)$ disjoint from $E(G)$ such that $(G \cup F, T)$ is planar, every cluster of $(G \cup F, T)$ is connected, and the edges in $F$ can be embedded so that every cluster of $(G \cup F, T)$ is in the outer face of every other cluster. We have the following simple fact regarding saturators already observed by Feng, Cohen and Eades [14].

Observation 1. An embedded flat clustered graph $(G, T)$ is c-planar if and only if $(G, T)$ has a saturator.

In order to model our problem by matroids we need to avoid two saturating edges in one face coming from two different clusters (even if they do not cross). In general, this is not possible if the face is not a simple cycle. Thus, we first augment the graph by adding some edges inside the faces.

Lemma 3. An embedded flat clustered graph $(G, T)$, all of whose faces are incident to at most five vertices, can be augmented by adding edges into an embedded flat clustered graph $\left(G^{\prime}, T^{\prime}\right)$ such that $(G, T)$ is c-planar if and only if $\left(G^{\prime}, T^{\prime}\right)$ is c-planar, and the following holds for $\left(G^{\prime}, T^{\prime}\right)$. If $\left(G^{\prime}, T^{\prime}\right)$ is c-planar then $\left(G^{\prime}, T^{\prime}\right)$ has a saturator $F$ whose edges can be embedded so that each face of $G^{\prime}$ contains at most one edge of $F$. 


\section{Proof of Theorem 3}

We give an algorithm for deciding c-planarity for embedded flat clustered graphs satisfying the hypothesis of the claim. By an algorithmic version of Lemma 3, from the given embedded flat clustered graph $(G, T)$ we obtain a new embedded graph $\left(G^{\prime}, T^{\prime}\right)$ such that every minimal saturator of $\left(G^{\prime}, T^{\prime}\right)$ has at most one edge inside each face and $\left(G^{\prime}, T^{\prime}\right)$ is c-planar if and only if $(G, T)$ is c-planar. This can be done easily in linear time in the number of vertices. Thus, it is enough to show that we can decide c-planarity of $\left(G^{\prime}, T^{\prime}\right)$ in polynomial time.

By Observation 1, it is enough to decide whether we can saturate $G^{\prime}$ so that all the clusters are connected and every cluster is drawn in the outer face of every other cluster. The latter can be tested in quadratic time in the number of vertices. In order to test the existence of a saturator we define two matroids for which we will use the matroid intersection algorithm. The ground set of each matroid is the multiset $\overline{E^{\prime}}$ of non-edges of $G^{\prime}$ defined as the union $\bigcup_{f} E_{f}$, over faces of $G^{\prime}$, where $E_{f}$ is the set of diagonals of the face $f$.

The first matroid, $M_{1}$, is the direct sum of graphic matroids constructed for each cluster. More precisely, denote the clusters by $C_{i}, i=1, \ldots, k$, and let $v \sim_{i} u$ if $u$ and $v$ are connected in $G^{\prime}\left[C_{i}\right]$. Denote by $G_{i}$ the multigraph obtained from $\overline{G^{\prime}}=$ $\left(V, \overline{E^{\prime}}\right)$ by deleting the vertices not in $C_{i}$, contracting the $\sim_{i}$-equivalent vertices into new vertices, and deleting all loops. Now, the ground set of the graphic matroid $M\left(G_{i}\right)$ can be identified with the set of edges from $\overline{E^{\prime}}$ that go between two vertices from $C_{i}$ belonging to distinct connected components of $C_{i}$. The rank of $M\left(G_{i}\right)$ is the number of vertices of $G_{i}$ minus one. Since the matroids $M\left(G_{i}\right), i=1, \ldots, k$, are pairwise disjoint, their direct sum, $M_{1}$, is also a matroid and its rank is the sum of the ranks of $M\left(G_{i}\right)$-s. The second matroid, $M_{2}$, is a partition matroid. A subset of $\overline{E^{\prime}}$ is independent in $M_{2}$ if it has at most one edge in every face of $G^{\prime}$.

Let $M$ be the intersection of $M_{1}$ and $M_{2}$. If $M$ has the same rank as $M_{1}$ then there exists a saturator of $\left(G^{\prime}, T^{\prime}\right)$ that has at most one edge inside each face. Thus, $\left(G^{\prime}, T^{\prime}\right)$ is c-planar by Observation 1 and that in turn implies that $(G, T)$ is c-planar as well. On the other hand, if $(G, T)$, and hence $\left(G^{\prime}, T^{\prime}\right)$, is c-planar then there exists a minimal saturator $F$ of $G^{\prime}$ that has at most one edge inside each face by the property of $G^{\prime}$ guaranteed by Lemma 3 Thus, $F$ witnesses the fact that the rank of $M_{1}$ and the rank of $M$ are the same. Hence, $M$ has the same rank as $M_{1}$ if and only if $\left(G^{\prime}, T^{\prime}\right)$ is c-planar and the theorem follows by the matroid intersection algorithm.

\section{Concluding Remarks}

By the construction in Section 4 we cannot hope for the fully general variant of the Hanani-Tutte theorem for clustered graphs. Nevertheless, it is still interesting to ask whether the weak or the strong Hanani-Tutte theorem for the case of flat clustered graphs holds if the graph obtained by contracting the clusters is acyclic (after deleting loops and multiple edges). More formally, given a flat clustered graph $(G, T)$, let $G_{T}$ denote the simple graph whose vertices correspond to clusters of $(G, T)$ and two distinct vertices $\mu$ and $\nu$ are joined by an edge if and only if there exists an edge in $G$ between the clusters $V(\mu)$ and $V(\nu)$. 
Conjecture 1. If $G_{T}$ is acyclic and $(G, T)$ admits an independently even clustered drawing then $(G, T)$ is c-planar.

A variant of the conjecture when $G_{T}$ is a path would provide a polynomial time algorithm for c-planarity testing for strip clustered graph, which is an open problem stated in [1]. Note that our proof from Section 5 fails if the graph has hexagonal faces. We wonder if this difficulty can be overcome or rather could lead to NP-hardness.

Acknowledgements. We are grateful to the numerous anonymous reviewers for many valuable comments.

\section{References}

1. Angelini, P., Da Lozzo, G., Di Battista, G., Frati, F.: Strip planarity testing. In: Wismath, S., Wolff, A. (eds.) GD 2013. LNCS, vol. 8242, pp. 37-48. Springer, Heidelberg (2013)

2. Biedl, T.C.: Drawing planar partitions III: Two constrained embedding problems. Technical report, RUTCOR, Rutgers University (1998)

3. Cairns, G., Nikolayevsky, Y.: Bounds for generalized thrackles. Discrete Comput. Geom. 23(2), 191-206 (2000)

4. Chimani, M., Zeranski, R.: Upward planarity testing: A computational study. In: Wismath, S., Wolff, A. (eds.) GD 2013. LNCS, vol. 8242, pp. 13-24. Springer, Heidelberg (2013)

5. Cortese, P.F., Di Battista, G.: Clustered planarity (invited lecture). In: Twenty-First Annual Symposium on Computational Geometry (Proc. SoCG 2005), pp. 30-32. ACM (2005)

6. Cortese, P.F., Di Battista, G., Frati, F., Patrignani, M., Pizzonia, M.: C-planarity of cconnected clustered graphs. J. Graph Algorithms Appl. 12(2), 225-262 (2008)

7. Cortese, P.F., Di Battista, G., Patrignani, M., Pizzonia, M.: Clustering cycles into cycles of clusters. J. Graph Algorithms Appl. 9(3), 391-413 (2005)

8. Cunningham, W.H.: Improved bounds for matroid partition and intersection algorithms. SIAM Journal on Computing 15(4), 948-957 (1986)

9. de Fraysseix, H., de Mendez, P.O., Rosenstiehl, P.: Trémaux trees and planarity. International Journal of Foundations of Computer Science 17(05), 1017-1029 (2006)

10. de Fraysseix, H., Rosenstiehl, P.: A characterization of planar graphs by Trémaux orders. Combinatorica 5(2), 127-135 (1985)

11. Di Battista, G., Frati, F.: Efficient c-planarity testing for embedded flat clustered graphs with small faces. In: Hong, S.-H., Nishizeki, T., Quan, W. (eds.) GD 2007. LNCS, vol. 4875, pp. 291-302. Springer, Heidelberg (2008)

12. Edmonds, J.: Submodular functions, matroids, and certain polyhedra. In: Jünger, M., Reinelt, G., Rinaldi, G. (eds.) Combinatorial Optimization - Eureka, You Shrink! LNCS, vol. 2570, pp. 11-26. Springer, Heidelberg (2003)

13. Feng, Q.-W., Cohen, R.F., Eades, P.: How to draw a planar clustered graph. In: Li, M., Du, D.-Z. (eds.) COCOON 1995. LNCS, vol. 959, pp. 21-30. Springer, Heidelberg (1995)

14. Feng, Q.W., Cohen, R.F., Eades, P.: Planarity for clustered graphs. In: Spirakis, P.G. (ed.) ESA 1995. LNCS, vol. 979, pp. 213-226. Springer, Heidelberg (1995)

15. Fulek, R.: Towards Hanani-Tutte theorem for clustered graphs. In: 40th International Workshop on Graph-Theoretic Concepts in Computer Science (accepted)

16. Fulek, R., Kynčl, J., Malinović, I., Pálvölgyi, D.: Efficient c-planarity testing algebraically. arXiv:1305.4519 
17. Fulek, R., Pelsmajer, M., Schaefer, M., Štefankovič, D.: Hanani-Tutte, monotone drawings and level-planarity. In: Pach, J. (ed.) Thirty Essays in Geometric Graph Theory, pp. 263-288 (2012)

18. Gall, F.L.: Powers of tensors and fast matrix multiplication. CoRR abs/1401.7714 (2014)

19. Gutwenger, C., Jünger, M., Leipert, S., Mutzel, P., Percan, M., Weiskircher, R.: Advances in c-planarity testing of clustered graphs. In: Goodrich, M.T., Kobourov, S.G. (eds.) GD 2002. LNCS, vol. 2528, pp. 220-236. Springer, Heidelberg (2002)

20. Gutwenger, C., Mutzel, P., Schaefer, M.: Practical experience with Hanani-Tutte for testing c-planarity. In: 2014 Proceedings of the Sixteenth Workshop on Algorithm Engineering and Experiments (ALENEX), pp. 86-97 (2014)

21. Hanani, H.: Über wesentlich unplättbare Kurven im drei-dimensionalen Raume. Fundamenta Mathematicae 23, 135-142 (1934)

22. Hong, S., Nagamochi, H.: Two-page book embedding and clustered graph planarity. Technical report, Dept. of Applied Mathematics and Physics, University of Kyoto (2009)

23. Hopcroft, J., Tarjan, R.: Efficient planarity testing. J. ACM 21(4), 549-568 (1974)

24. Ibarra, O.H., Moran, S., Hui, R.: A generalization of the fast LUP matrix decomposition algorithm and applications. J. Algorithms 3(1), 45-56 (1982)

25. Katz, B., Rutter, I., Woeginger, G.: An algorithmic study of switch graphs. In: Paul, C., Habib, M. (eds.) WG 2009. LNCS, vol. 5911, pp. 226-237. Springer, Heidelberg (2010)

26. Kuratowski, K.: Sur le problème des courbes gauches en topologie. Fund. Math. 15, 271-283 (1930)

27. Lawler, E.L.: Matroid intersection algorithms. Mathematical Programming 9, 31-56 (1975)

28. Lengauer, T.: Hierarchical planarity testing algorithms. J. ACM 36(3), 474-509 (1989)

29. Oxley, J.: Matroid Theory. Oxford University Press (2011)

30. Pach, J., Tóth, G.: Which crossing number is it anyway? J. Combin. Theory Ser. B 80(2), 225-246 (2000)

31. Pach, J., Tóth, G.: Monotone drawings of planar graphs. J. Graph Theory 46(1), 39-47 (2004), updated version: arXiv:1101.0967

32. Pelsmajer, M.J., Schaefer, M., Stasi, D.: Strong Hanani-Tutte on the projective plane. SIAM Journal on Discrete Mathematics 23(3), 1317-1323 (2009)

33. Pelsmajer, M.J., Schaefer, M., Štefankovič, D.: Removing even crossings. J. Combin. Theory Ser. B 97(4), 489-500 (2007)

34. Pelsmajer, M.J., Schaefer, M., Štefankovič, D.: Removing even crossings on surfaces. European Journal of Combinatorics 30(7), 1704-1717 (2009)

35. Schaefer, M.: Hanani-Tutte and related results. To appear in Bolyai Memorial Volume

36. Schaefer, M.: Toward a theory of planarity: Hanani-tutte and planarity variants. J. Graph Algorithms Appl. 17(4), 367-440 (2013)

37. Tutte, W.T.: Toward a theory of crossing numbers. J. Combin. Theory 8, 45-53 (1970)

38. Whitney, H.: Non-separable and planar graphs. Trans. Amer. Math. Soc. 34, 339-362 (1932)

39. Williams, V.V.: Multiplying matrices faster than Coppersmith-Winograd. In: Proceedings of the Forty-Fourth Annual ACM Symposium on Theory of Computing, STOC 2012, pp. 887898 (2012) 